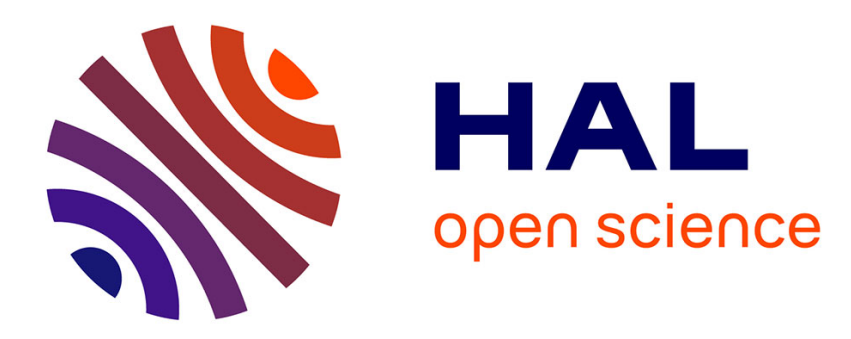

\title{
Diffusional relaxation in a random sequential adsorption model
}

\author{
B. Bonnier
}

\section{To cite this version:}

B. Bonnier. Diffusional relaxation in a random sequential adsorption model. Physical Review E , 1997, 56 (6), pp.7304-7305. 10.1103/PhysRevE.56.7304 . hal-01566062

\section{HAL Id: hal-01566062 \\ https://hal.science/hal-01566062}

Submitted on 5 Dec 2018

HAL is a multi-disciplinary open access archive for the deposit and dissemination of scientific research documents, whether they are published or not. The documents may come from teaching and research institutions in France or abroad, or from public or private research centers.
L'archive ouverte pluridisciplinaire HAL, est destinée au dépôt et à la diffusion de documents scientifiques de niveau recherche, publiés ou non, émanant des établissements d'enseignement et de recherche français ou étrangers, des laboratoires publics ou privés. 


\title{
Diffusional relaxation in a random sequential adsorption model
}

\author{
B. Bonnier* \\ Centre de Physique Théorique et de Modélisation, URA 1537 CNRS, Université Bordeaux I, 19 Rue du Solarium, \\ 33175 Gradignan Cedex, France
}

(Received 7 July 1997)

\begin{abstract}
The one-dimensional random sequential adsorption dimer model with diffusional relaxation is revisited using the numerical and perturbative results recently obtained by C. K. Gan and J. S. Wang [Phys. Rev. E 55, 107 (1997)]. These results are shown to support the analytic expression that we derive for the large time behavior of the coverage and allow us to propose its modelization for any time and diffusion rate. [S1063-651X(97)00412-1]
\end{abstract}

PACS number(s): 05.70.Ln, 82.20.Mj, 05.50.+q

Random sequential adsorption (RSA) is a model in which objects are deposited on a given substrate one at a time, with random positions, such that new objects cannot overlap previously adsorbed ones [1]. A variety of large molecules adsorbs in an essentially irreversible manner, and the RSA model may be appropriate [2] to describe this process. Its asymptotic behavior at large time is then dominated by the formation of vacancies resulting in the jamming of the available area. When the deposited particles are subject to diffusion, as is required in some experimental situations [3], the coverage $\theta$ is expected to reach its closest-packing limit through a diffusive power-law approach. In one dimension, this reads $\Theta \simeq 1-C / \sqrt{t}$, as proven in the context of the diffusive monomer model [4], or supported by analytical arguments [5,6] and Monte Carlo (MC) simulations [5,7] for the diffusive dimer model that is unsolved and that we study here.

This model, introduced by Privman and Nielaba [5], is defined as follows. One starts with an empty infinite onedimensional lattice. The dynamics involve picking two neighboring sites randomly and then deciding either to make a deposition with probability $p$ or to make a diffusion to the left or right with equal probabilities $(1-p) / 2$. If the chosen attempt is impossible, one starts over, picking two new sites. We have already [6] studied this model and proposed the asymptotic expression $\Theta \simeq 1-\sqrt{p / 16 \pi(1-p) t}$ on the basis of the MC data of Ref. [5]. Our approach is motivated by the results recently obtained [7] for the density of empty sites $\rho(\gamma, t)=1-\Theta$ at time $t, \gamma$ being the relative rate $\gamma=(1$ $-p) / 2 p$. Its series expansion in powers of $t$, where each term is a function of $\gamma$, has been derived up to order 31 and extensive MC simulations have been performed up to large time $\left(t \simeq 10^{6}\right)$ for 8 values of $\gamma$ ranging from 0.05 to 6.4 . From these results, it is concluded in the work of Ref. [7] that the MC data confirm the asymptotic behavior $\rho(\gamma, t)$ $\simeq C(\gamma) / \sqrt{t}$ but that it appears impossible to derive it from the series analysis, whose range of applicability seems limited to $0 \leqslant t \lesssim 10$, i.e., one or two orders of magnitude below the diffusive asymptotic regime. In addition, the MC data indicate a scaling law $\rho(\gamma, t)=F(t / \gamma) / \gamma$, but the function $F(z)$ is not specified.

\footnotetext{
*Electronic address: Bonnier@bortibm1.in2p3.fr
}

This situation can be improved, as shown here: we propose an analytic expression for the scaling function and a parametrization of the density from its series expansion, which appears to be quite accurate for any values of $\gamma$ and $t$.

As for the derivation of $F(z)$, we repeat the arguments of our previous work [6], which are expected to hold in case of rapid deposition (small $\gamma$ ), and show that the result agrees for all the values of the rate $\gamma$ with the MC data. (This kind of argument has been recently applied to the diffusive monomer model [8].) At long times, there remains only isolated vacant sites $A$, their disappearance being limited by the necessity to form pairs $A A$, which can be filled by a dimer deposition. If the deposition is rapid, the system thus behaves like the diffusion-limited reaction $A+A \rightarrow$ inert. In this model the density of surviving particles $A$, which asymptotically approaches $\rho(\gamma, t)$, can be exactly solved [9] and becomes $\rho(\gamma, t)=2 \rho\left(\gamma, t_{0}\right) \int_{\Lambda}^{\infty} \exp \left(\Lambda^{2}-x^{2}\right) d x / \sqrt{\pi}$, for large time $t$ $\geqslant t_{0}$, where in the normalization of Ref. [7] $\Lambda$ $=4 \rho\left(\gamma, t_{0}\right) \sqrt{2 \gamma\left(t-t_{0}\right)}$. Taking the leading term in the asymptotic expansion of this expression, which is independent of $\rho\left(\gamma, t_{0}\right)$, one obtains $\rho(\gamma, t) \simeq 1 / \sqrt{32 \pi \gamma t}$, i.e., $F(z)$ $=1 / \sqrt{32 \pi z}$. In order to check this behavior, we take the MC data of Ref. [7] for the largest value $t_{\infty}=10^{6}$ where the asymptotic regime is reached and compute the quantity $C_{\infty}$ defined as $C_{\infty}=-\ln \left[\rho\left(\gamma, t_{\infty}\right) \sqrt{\gamma t_{\infty}}\right]$, that we expect to be $C_{\infty}$ $\simeq \ln (\sqrt{32 \pi})=2.305$. The results of this analysis for all the available values of the rate $\gamma$ are depicted in the first lines of Table I where it appears that, within the experimental precision, $C_{\infty}$ has its predicted value, even for the largest rates.

We now consider the determination of $\rho(\gamma, t)$ on the whole time range from its series expansion. This is difficult when the density becomes significantly smaller than its asymptotic value $e^{-2}$ reached in a pure RSA process $(\gamma=0)$, i.e., for $t \gtrsim 10$ according to Ref. [7]. We thus take into account, in addition to our knowledge [1] of the density in the RSA process $\rho(\gamma=0, t)=\exp \{2[\exp (-t)-1]\}$, its behavior in the fast diffusive regime as solved in Ref. [10]. The density cannot be expressed as a simple function of time but one can show that if for any value of the rate one defines $\delta(\gamma, t)$ as $\delta(\gamma, t)=\rho(\gamma, t)\{1+4 t+\ln [\rho(\gamma, t)]\}$ it fulfills the relation $\delta(\gamma=\infty, t)=1$. We finally consider the function $\Sigma(\gamma, t)=\{[\delta(\gamma, t)-1](1+\sqrt{1+2 \gamma t})\} / 2[\delta(0, t)-1]$ whose series expansion is known from the series of $\rho(\gamma, t)=1$ 
TABLE I. For the values of $\gamma$ given on top of each column, the MC values of $-\ln \rho(\gamma, t)$ taken from Ref. [7] are given line $2\left(t=t_{\infty}\right.$ $\left.=10^{6}\right)$ and line $4\left(t=t_{1}=100\right)$. The data of line 2 are used to compute the quantity $C_{\infty}$ defined as $C_{\infty}=-\ln \left[\rho\left(\gamma, t_{\infty}\right) \sqrt{\gamma t_{\infty}}\right]$ given line 3 and whose theoretically expected value is 2.305 . The data of line 4 have to be compared to our results given line 5 (line Padé), obtained from the series analysis described in the text and which gives for $\Sigma(\gamma, t=\infty)$ the values given line 6 , its theoretically expected value being 0.521 .

\begin{tabular}{|c|c|c|c|c|c|c|c|c|}
\hline$\gamma$ & 0.05 & 0.1 & 0.2 & 0.4 & 0.8 & 1.6 & 3.2 & 6.4 \\
\hline$-\ln \left[\rho\left(\gamma, t_{\infty}\right)\right]$ & 7.71 & 8.06 & 8.41 & 8.76 & 9.11 & 9.45 & 9.79 & 10.13 \\
\hline$C_{\infty}$ & 2.30 & 2.30 & 2.31 & 2.31 & 2.31 & 2.31 & 2.30 & 2.30 \\
\hline$-\ln \left[\rho\left(\gamma, t_{1}\right)\right]$ & 3.21 & 3.49 & 3.80 & 4.10 & 4.40 & 4.75 & 4.95 & 5.15 \\
\hline Padé & 3.21 & 3.51 & 3.81 & 4.07 & 4.27 & 4.65 & 4.78 & 5.28 \\
\hline$\Sigma(\gamma, t=\infty)$ & 0.58 & 0.55 & 0.53 & 0.54 & 0.55 & 0.51 & 0.58 & 0.35 \\
\hline
\end{tabular}

$-2 t+3 t^{2}-\frac{11}{3} t^{3}+\frac{47}{12} t^{4}-\frac{1}{120}(454+8 \gamma) t^{5}+\cdots$ given in Ref. [7] at order 31 . It reads $\Sigma(\gamma, t)=1+\frac{1}{10} \gamma t+\cdots$, i.e., a simpler and shorter one (its order is 27). Our aim is to reconstruct $\Sigma(\gamma, t)$ from this expansion and for its resummation we simply construct its $[N, N]$ diagonal Padé approximants $P_{N}(\gamma, t)$ up to $N=13$. This is motivated by the observation that $\Sigma(\gamma, t=\infty)$ must be finite due to the asymptotic behavior of the density previously described. In fact, inserting in the definition of $\Sigma(\gamma, t)$ the asymptotic behavior of the density, one gets $\Sigma(\gamma, t=\infty)=e^{2} / 8 \sqrt{\pi} \simeq 0.521$ for any positive rate. In the last line of the Table I, we give the asymptotic value of the $[12,12]$ approximant for various rates (the $[13,13]$ is in general comparable, but does not improve the results), which in general compare well with this prediction. For any finite value $t_{1}$ of the time, we obtain the numerical value of the density $\rho\left(\gamma, t_{1}\right)$ as follows. The value of $\Sigma\left(\gamma, t_{1}\right)$ is provided by its approximants $P_{N}\left(\gamma, t_{1}\right)$ and it thus gives $\delta\left(\gamma, t_{1}\right)$ as we take for $\delta\left(0, t_{1}\right)$ its exact value. Finally, $\delta\left(\gamma, t_{1}\right)$ gives the density. We give our results (obtained with $N=12$ ) in Table I for the value $t_{1}=100$, as an example of a large but preasymptotic value, and one can check their general agreement with the MC data. When $t_{1}$ decreases, the agreement increases.

In conclusion, we want to point out that it is possible, from the results of Ref. [7], to derive a reasonably good phenomenological description of the diffusive monomer model in the general case.
[1] M. C. Bartelt and V. Privman, Int. J. Mod. Phys. B 5, 2883 (1991); J. W. Evans, Rev. Mod. Phys. 65, 1281 (1993).

[2] J. Feder, J. Theor. Biol. 87, 237 (1980); B. Senger, P. Schaaf, F. J. Bafaluy, F. J. G. Cuisinier, J. Talbot, and J. C. Voegel, Proc. Natl. Acad. Sci. USA 91, 3004 (1994).

[3] J. J. Ramsden, J. Stat. Phys. 73, 853 (1993).

[4] M. D. Grynberg, T. J. Newman, and R. B. Stinchcombe, Phys. Rev. E 50, 957 (1994); M. D. Grynberg and R. B. Stinch- combe, Phys. Rev. Lett. 74, 1242 (1995).

[5] V. Privman and P. Nielaba, Europhys. Lett. 18, 673 (1992).

[6] B. Bonnier and J. McCabe, Europhys. Lett. 25, 399 (1994).

[7] C. K. Gan and J. S. Wang, Phys. Rev. E 55, 107 (1997).

[8] E. Eisenberg and A. Baram, J. Phys. A 30, L271 (1997).

[9] D. C. Torney and H. M. McConnel, J. Phys. Chem. 87, 1941 (1983).

[10] V. Privman and M. Barma, J. Chem. Phys. 97, 6714 (1992). 